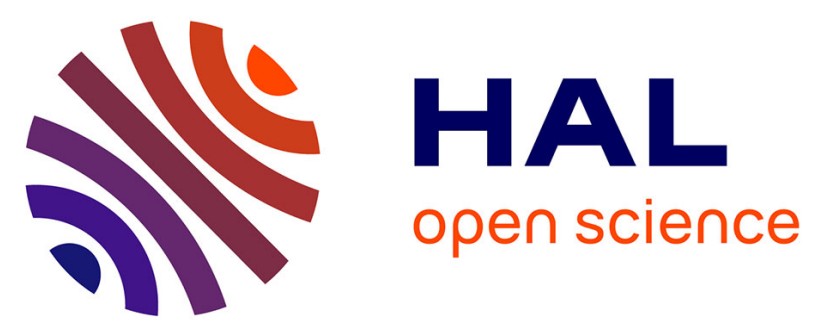

\title{
Vibrational response of free standing single copper nanowire through transient reflectivity microscopy
}

Laurent Belliard, Thomas W. Cornelius, Bernard Perrin, Nazim Kacemi, Loic

Becerra, Olivier Thomas, Maria Eugenia Toimil-Molares, Marco Cassinelli

\section{- To cite this version:}

Laurent Belliard, Thomas W. Cornelius, Bernard Perrin, Nazim Kacemi, Loic Becerra, et al.. Vibrational response of free standing single copper nanowire through transient reflectivity microscopy. Journal of Applied Physics, 2013, 114 (19), pp.193509. 10.1063/1.4831957 . hal-01233814

\section{HAL Id: hal-01233814 https://hal.science/hal-01233814}

Submitted on 11 Oct 2018

HAL is a multi-disciplinary open access archive for the deposit and dissemination of scientific research documents, whether they are published or not. The documents may come from teaching and research institutions in France or abroad, or from public or private research centers.
L'archive ouverte pluridisciplinaire HAL, est destinée au dépôt et à la diffusion de documents scientifiques de niveau recherche, publiés ou non, émanant des établissements d'enseignement et de recherche français ou étrangers, des laboratoires publics ou privés. 


\title{
Vibrational response of free standing single copper nanowire through transient reflectivity microscopy
}

\author{
Laurent Belliard, ${ }^{1, a)}$ Thomas W. Cornelius, ${ }^{2}$ Bernard Perrin, ${ }^{1}$ Nazim Kacemi, ${ }^{1}$ \\ Loïc Becerra, ${ }^{1}$ Olivier Thomas, ${ }^{2}$ Maria Eugenia Toimil-Molares, ${ }^{3}$ and Marco Cassinelli ${ }^{3}$ \\ ${ }^{1}$ Institut des NanoSciences de Paris, UMR7588 CNRS, Université Pierre et Marie Curie, 4 place Jussieu, \\ 75005 Paris, France \\ ${ }^{2}$ Aix-Marseille Université, CNRS, IM2NP UMR 7334, Faculté des Sciences, Campus de Saint-Jérôme, \\ Avenue Escadrille Normandie Niemen-Case 142, F-13397 Marseille Cedex, France \\ ${ }^{3}$ GSI Helmholtz Centre for Heavy Ion Research, Planckstr. 1, D-64291 Darmstadt, Germany
}

We report on the ultrafast vibrational response of single copper nanowires investigated by femtosecond transient reflectivity measurements. The oscillations of the sample reflectivity are correlated with individual modes of resonance for wires with a diameter ranging from 100 to $500 \mathrm{~nm}$ and are compared with 2D finite element simulation. Fluctuation of the sample-substrate coupling is illustrated through its effect on the damping rate. We demonstrate elastic confinement in free standing wires which allowed the detection of up to the third harmonic of the breathing mode. By removing the energy relaxation channel towards the substrate, we obtained nano-oscillators with quality factors up to 130. Finally, taking advantage of the very high spectral resolution achieved on free standing wires, we could observe the elastic coupling between two close wires via their polymer cladding.

\section{INTRODUCTION}

In nanoparticles, coherent acoustic vibrations may be excited by light absorption. Both the absorption, assisted by the well known localized surface plasmon phenomenon and the vibrational response, related to energy transfer towards the lattice, have been intensively studied during the last decades. Phonon as well as electron confinement in small particles give new opportunities in various fields as photonics, ${ }^{1}$ electronics, ${ }^{2}$ chemistry, ${ }^{3}$ or biology for cancer therapy. ${ }^{4,5}$ The vibrational resonances and the plasmon oscillations can be tuned by the size, the shape, and the local environment. Especially for vibrational modes in nanoscale systems, the eigenmodes exhibit frequencies in the range of a few $10 \mathrm{GHz}$ up to $1 \mathrm{THz}$. In order to address such high frequency features, time-resolved optical spectroscopy experiments are now considered as a powerful approach. Ultra-short femtosecond pump pulses induce a fast lattice heating by electron-phonon coupling. The associated thermal expansion is optically detected by a time delayed probe pulse. Initially, the measurements were performed on a large number of nanoparticles in order to increase the signal to noise ratio. Nevertheless, even if the synthesis processes are nowadays more and more controlled, the vibrational response is always strongly affected by the inherent size and shape distribution of the nanoparticle ensemble. In this case, it is not possible to obtain any accurate information about nanoparticles elastic properties.

In order to overcome this drawback, recent measurements on single nanoparticles have been reported. Since the

\footnotetext{
a) Author to whom correspondence should be addressed. Electronic mail: laurent.belliard@upmc.fr
}

pioneer work of van Dijk et al., ${ }^{6}$ which investigated the dynamic response of gold nanospheres, a large variety of materials and particle shape has been investigated like nanoprisms, ${ }^{7}$ nanowires, ${ }^{8}$ nanorings, ${ }^{9}, 10$ nanocubes, ${ }^{11}$ nanorods, ${ }^{12}$ dimer nanoparticules, ${ }^{13}$ and nanoparticles. ${ }^{14}$ All these results have been obtained by measuring the transient absorption signature, in transmission geometry using high numerical aperture objective to recollect a maximum of light. Moreover, few results have been recently reported on reflectivity configuration in near ${ }^{15-17}$ and far field. ${ }^{18-20}$ In general, the vibrational modes observed are assigned to breathing modes of the structure. According to the particle shape, additional extensional or flexural signatures have been also reported. ${ }^{7,12,21}$ The common denominator of all these previous studies is the clear evidence that particle coupling with the local environment leads to a huge damping rate. The particle-substrate interaction is at the origin of the acoustic energy transfer into the substrate. As a direct consequence, the resonator exhibits a low quality factor, defined by the energy stored over the energy lost in one cycle. Nevertheless, such an interaction has been recently tuned in order to create an efficient bulk transverse wave emitter with tunable frequency. ${ }^{22}$ In order to get insight about the energy relaxation process, across the particle surface, different routes have been investigated. In the case of particles embedded in a glass matrix, the increasing hydrostatic pressure obtained with the diamond anvil cell technol$\mathrm{ogy}^{23}$ has demonstrated a huge effect on the damping rates. Additionally, the lifetime of nanoring vibrations has been reduced by immersion in glycerol solution. ${ }^{9}$ In both cases, the opening of additional relaxation channels contributes to reduce the vibrational spectral richness of the nanoparticles. Indeed, even if the measurements are performed on a single nanoparticle, broad peaks are revealed on the Fourier 
spectrum, and thus a fine estimation of the elastic properties is very challenging.

To assess the validity of the classical continuum theories at the nanoscale,,$^{5,24}$ and to enable a high quality factor response, the elastic energy must be confined into the nanostructure and any mechanical coupling must be suppressed. Experimentally, this is achieved by investigating free standing nanostructures. In this work, the ultrafast vibrational response of single copper nanowires, with diameter ranging from $100 \mathrm{~nm}$ up to $500 \mathrm{~nm}$, has been investigated by femtosecond transient reflectivity measurements. The observed sample reflectivity oscillations are related to individual resonance modes. We compare time resolved acoustic signatures measured on a nanowire deposited on a (100) silicon wafer and those of its free standing counterpart. We demonstrate that a large elastic energy confinement gives the opportunity to observe up to the third harmonic of the fundamental breathing mode. Moreover, additional modes coming from a non uniform wire cladding have been identified. Finally, the realization of these high quality factor oscillators enables us to demonstrate an acoustic coupling effect between two nanowires located at a very short distance.

\section{EXPERIMENTAL DETAILS AND SAMPLE DESCRIPTION}

\section{A. Sample preparation}

Copper nanowires were prepared by electrodeposition in etched ion-track membranes. ${ }^{26}$ To fabricate the membranes, $30 \mu \mathrm{m}$ thick polycarbonate foils (MAKROFOL, Bayer) were irradiated at the UNILAC accelerator of GSI with $\sim \mathrm{GeV}$ $\mathrm{Au}^{25+}$ ions. Each ion creates a damaged cylindrical zone along its trajectory through the foil called latent track. After irradiation, these tracks were selectively etched in a $6 \mathrm{M}$ $\mathrm{NaOH}$ solution at $50^{\circ} \mathrm{C}$ resulting in cylindrical smooth nanochannels. The channel diameter increases nearly linearly with the etching time. After etching, the membranes were rinsed in deionized water. We then sputtered a thin layer of $\mathrm{Au}$ on one side of the membrane. The layer was reinforced electrochemically with $\mathrm{Cu}$, and served as cathode in a two-electrode electrochemical cell, while a $\mathrm{Cu}$ rod served as anode. After nanowire growth, the polymer membrane was dissolved in dichloromethane, and the wires were detached from the cathode by ultrasonification. In a final step, the nanowires were drop-casted onto the substrates.

In order to reduce the influence of the silicon substrate on the dynamic response, we dispersed the wires on a Si wafer which has been preliminary structured with periodic trenches (Figure 1). We used a 2 in. n-type $\mathrm{Si}(001)$ substrate. First, a dry oxidation using oxygen gas in a tube furnace leads to the formation of ${\mathrm{a} \mathrm{SiO}_{2}}_{2}$ layer at the $\mathrm{Si}$ wafer surface. A positive resist (Shipley S1813) was spin coated on the surface and exposed to UV light using a standard optical lithography system. The key point in this process is to take care of the alignment of the desired structures with respect to the wafer's flat. After being developed, rinsed in water, blown dry, and postbaked, the sample was etched. $\mathrm{SiO}_{2}$ was locally stripped in a buffered HF solution (BHF) and resist was removed in acetone. Finally, the sample with a patterned

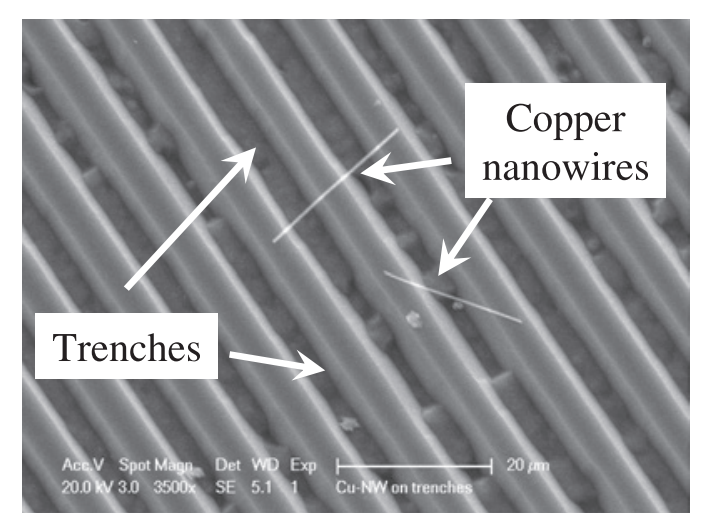

FIG. 1. SEM image showing two single copper nanowires placed across pyramidal trenches fabricated by lithography and anisotropic silicon etching.

oxide mask on top was dipped in a hot $40 \% \mathrm{KOH}$ solution to perform anisotropic Si etching. This process, resulting in pits of pyramidal form, was stopped when the trenches reached $\sim 2 \mu \mathrm{m}$ depth. The last step of the patterning substrate method consisted in removing with $\mathrm{BHF}$ the remaining $\mathrm{SiO}_{2}$ layer from the surface yielding the desired trench network in the silicon wafer. The trenches are aligned along the $\langle 110\rangle$ direction, with a periodicity close to $10 \mu \mathrm{m}$ (Figure 1).

\section{B. Experimental setup}

Our experimental setup uses a mode-locked Ti:sapphire (MAI-TAI Spectra) laser source operating at $800 \mathrm{~nm}$, with a repetition rate of $79.8 \mathrm{MHz}$. The pulse time width is less than $100 \mathrm{fs}$ at the laser output. The pump beam is modulated at $1.8 \mathrm{MHz}$ to perform synchronous detection on the sample reflectivity. The pump and probe beams are focalized by an objective with a $\mathrm{NA}=0.9$ fixed on an $\mathrm{XY}$ piezoelectric stage. The objective lens is scanned over the surface to locate the wire. Then a measurement area is selected on the wire. The spatial resolution of such mapping along the wire is estimated to be $\sim 0.7 \mu \mathrm{m}$. In the present study, even if we can exalt the pump absorption process, we do not try to tune the pump wavelength close to the localized surface plasmon resonance which is strongly size dependent. ${ }^{27}$ To avoid scattered light coming from the pump beam, a two-color experiment is performed by doubling the pump frequency $(\lambda=400 \mathrm{~nm})$ with a nonlinear crystal. A dichroic filter located in front of the diode system suppresses the light of the pump beam. In order to prevent nanowire being damaged by the probe beam, its power is reduced below $50 \mu \mathrm{W}$ and the power of the pump beam does not exceed $200 \mu \mathrm{W}$. With such experimental conditions, the acoustic signal and the optical reflectivity are stable during all the average processing.

The reflectivity from the sample is measured by an avalanche detector and analyzed with a lock-in amplifier. A maximum pump-probe time delay equal to $12 \mathrm{~ns}$ is achieved using a mobile reflector system mounted on a translation stage.

\section{RESULTS AND DISCUSSION}

\section{A. Supported nanowires}

First, let us consider the case of a single $\mathrm{Cu}$ wire deposited on a flat (100) silicon wafer. Figure 2 presents a typical 


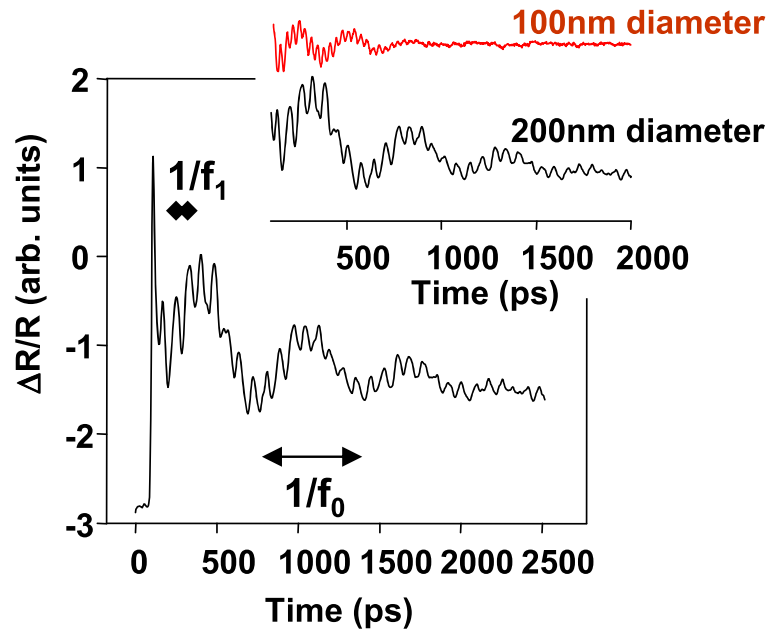

FIG. 2. (a) Transient sample reflectivity obtained on a single $200 \mathrm{~nm}$ diameter copper nanowire placed on a flat Si substrate. After the sharp electronic signal, we observe an oscillatory signature with two frequency components. The feature at lower frequencies, called $\mathrm{f}_{0}$, results from the contact with the substrate, while the feature at higher frequency, called $\mathrm{f}_{1}$, corresponds to the first breathing mode of the wire. (b) Time resolved measurements for copper wires with diameter 100 (red line) and $200 \mathrm{~nm}$ (black line).

change of reflectivity $(\Delta R / R)$ measured during $2.5 \mathrm{~ns}$ on a $200 \mathrm{~nm}$ diameter wire. The signal represents the vibration of the nanowire, which is clearly composed by two frequencies, a low frequency $f_{0}$ around $1.85 \mathrm{GHz}$ and a higher one $f_{1}$ around $15.6 \mathrm{GHz}$. The same behavior has been observed for thinner wires with a diameter of $100 \mathrm{~nm}$ (see Fig. 2, inset). The position of the two frequencies component is correlated to the particle size. Thus, it is blueshifted for smaller diameters. The evolution of $f_{1}$ has been followed for wires with a diameter ranging from 100 to $500 \mathrm{~nm}$ and compared with the frequency of the first breathing mode for an infinite wire in Fig. 3. The experimental values are depicted by red symbols, while the frequencies of the modes for an infinite wire are presented by empty black symbols. The latter may be deduced from the resolution of the propagation equation in cylindrical coordinates,

$$
\mu \nabla^{2} \vec{u}+(\lambda+\mu) \nabla \nabla \cdot \vec{u}=\rho\left(\partial^{2} \vec{u} / \partial t^{2}\right),
$$

where $\vec{u}$ is the displacement vector, $\rho$ the density, and $\lambda$ and $\mu$ the Lamé's constants.

The standard resolution is based on the Lamé potentials method for isotropic material ${ }^{28-30}$ and applying the free boundaries conditions. In the case of anisotropic systems, 2D finite elements simulations may be performed to extract the vibrational landscape.

The excellent agreement between the experimental values and the simulation, assuming an isotropic elastic behavior, allows us to clearly identify the first breathing resonance. This resonance is characterized by a pure radial displacement with a nod located at the center, as schematically shown in the inset of Figure 3 . In the present case, the variation of the dielectric constant is the origin of the optical signature. To extract the lifetime of this first resonance, the data were fitted with a sum of exponentially damped cosine function,

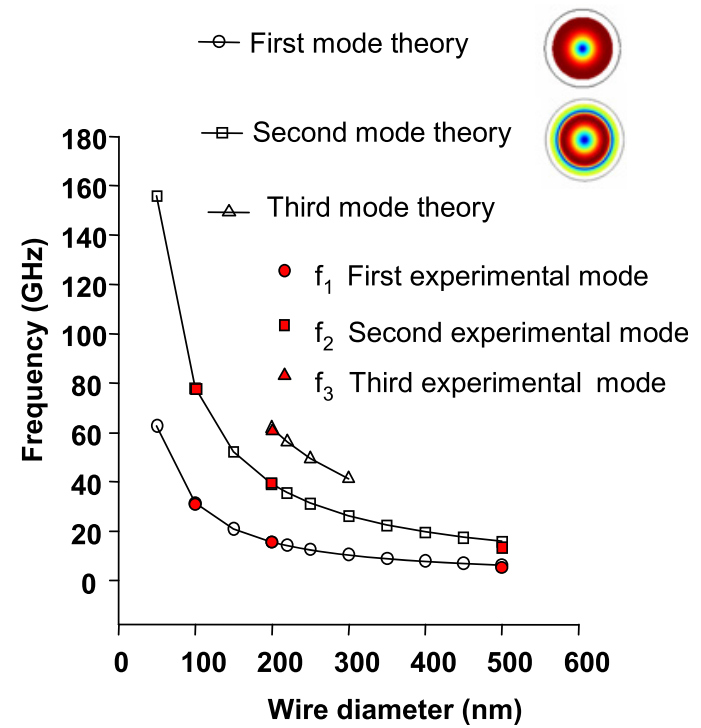

FIG. 3. Evolution of the frequency position of the first $f_{1}$, second $f_{2}$, and third $\mathrm{f}_{3}$ breathing modes versus the wire diameter. The full and empty symbols correspond to theoretical and experimental values, respectively. We obtained a very good agreement assuming isotropic elasticity for the copper wire. X-ray diffraction measurements performed on the same system have confirmed a polycrystalline structure. The Young modulus, Poisson ratio, and density have been fixed in finite elements simulation to $110 \mathrm{GPa}, 0.35$ and $8700 \mathrm{~kg} / \mathrm{m}^{3}$, respectively. Inset: calculated displacements of the breathing modes. The first mode of vibration exhibits a nod in the center while the displacement exhibits two nods in the case of the second harmonic.

$$
\Delta R=\sum_{i} A_{i} \exp \left(-\frac{t}{\tau_{i}}\right) \cos \left(2 \pi f_{i} t+\phi_{i}\right)
$$

where $A_{i}$ is the amplitude, $\tau_{i}$ is the lifetime, $f_{i}$ is the eigenmode frequency, and $\phi_{\mathrm{i}}$ is the phase.

The quality factor $\mathrm{Q}=\pi \mathrm{f} \tau$ is estimated at 28 and 39 for wires with diameter $100 \mathrm{~nm}$ and $200 \mathrm{~nm}$, respectively. Such low quality factors have been often reported in experiments performed in a similar geometrical set up. ${ }^{8}$ Indeed, the contact between the particle and the substrate is an open channel of energy relaxation which leads to a huge damping rate of the first breathing mode of vibration.

Recently, experiments performed in reflectivity geometry have demonstrated that the low frequency component $\mathrm{f}_{0}$ could be treated with the Hertz contact theory. ${ }^{19,31}$ Because of the sudden expansion associated with the breathing resonance, the particle-substrate interface oscillates with frequency related to the contact stiffness. Such approach could explain why the frequency of this oscillation increased for smaller diameter wire, in good agreement with previous results reported on spherical particles. ${ }^{19}$ In such case, the transient signature may be explained across an interferometric scheme. ${ }^{20}$ Here, the uniaxial nanowire geometry offers an unique opportunity to investigate the acoustic signature on a same system at different points along the growth direction. Figure 4 presents a reflectivity measurement performed on the same supported system but at another location. The observable mode splitting of the fundamental breathing mode, revealed by a better spectral resolution, is linked to a specific nanowire configuration and will be discussed later. Nevertheless, in the associated Fourier spectrum, the low 


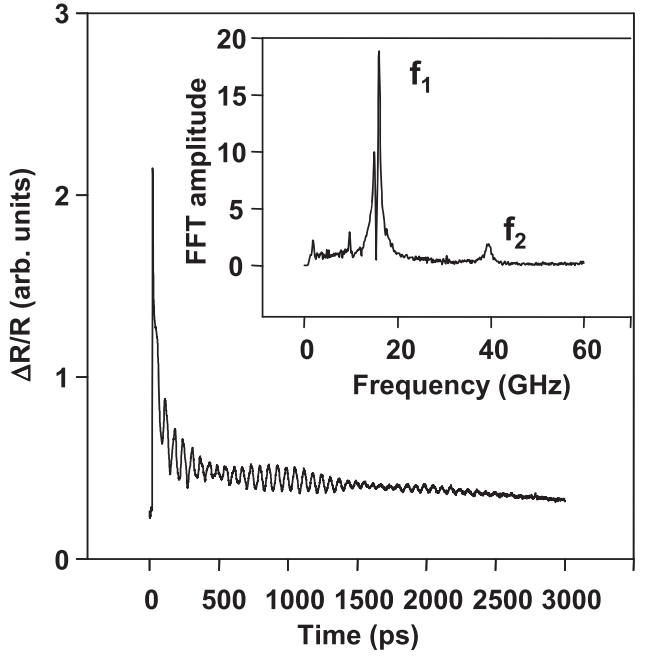

FIG. 4. Transient sample reflectivity obtained on a supported nanowire system composed by two closed nanowires. The observable splitting of the fundamental breathing mode linked to this specific configuration will be discussed at the end of this article. Nevertheless, we may observe a smaller damping rate, which may be related to a local modulation of particlesubstrate coupling amplitude. Inset: Fast Fourier transform showing a new acoustic feature $\mathrm{f}_{2}$ located around $39.6 \mathrm{GHz}$ which may be associated to the excitation of the second harmonic breathing mode.

frequency $\mathrm{f}_{0}$ has almost disappeared while a substantial increase of the quality factor for the first breathing mode has been determined now estimated at 50 . These two results indicate a local reduction of the contact between the nanowire and the substrate.

Moreover, the elastic confinement contributes to the detection of new vibrational features. Indeed, a higher frequency component is observed around $39.6 \mathrm{GHz}$. According to finite element simulations with different wire diameters, the evolution of this new signature reported in Fig. 3 fits very well with the expected behavior of the second order breathing mode, characterized by a pure radial displacement with two nods, (see inset Fig. 3).

Even if the fluctuation of the particle-substrate contact was fortuitous in the latter experiment, this result clearly demonstrates that the large dispersion of the damping rate reported in literature is linked to fluctuations of the boundaries conditions at the interface with the substrate. Additionally, we demonstrated that new vibrational eigenmodes could be detected if the substrate influence is reduced.

\section{B. Free standing nanowires}

In a next step, we aimed at the detection of higher vibrational modes by eliminating the influence of the substrate, placing the nanowires across microtrenches and investigating the vibrational landscape of a single free standing nanowire. Figure 5 presents the time resolved signature measured on a $200 \mathrm{~nm}$ diameter free standing $\mathrm{Cu}$ wire measured for longer delays $\sim 5 \mathrm{~ns}$. A clear oscillatory signature is thus revealed for the freestanding nanowire on a time scale considerably larger than the one of its counterpart wire placed on the $\mathrm{Si}$ surface ( $\sim 2-3 \mathrm{~ns}$, Fig. 2). As a direct consequence of the suppression of the relaxation channel towards the substrate, elastic confinement is increased. Now, the lifetime of the first and second breathing modes leads to quality factors equal to 80 and 130, respectively. Such increase of the life time has been recently observed in similar suspended gold nanowires. $^{32}$ In some cases, the signal to noise ratio is so high that the third order breathing mode located at $\sim 60 \mathrm{GHz}$ can be detected, the resonance frequency being in very good agreement with the simulations (see Fig. 3). The detection of high harmonic signature has to be emphasized. Indeed, atomistic simulations ${ }^{33}$ predicted that the breakdown of the elasticity may occur for bigger nanoparticles on the high harmonic rather than for fundamental modes. In general with
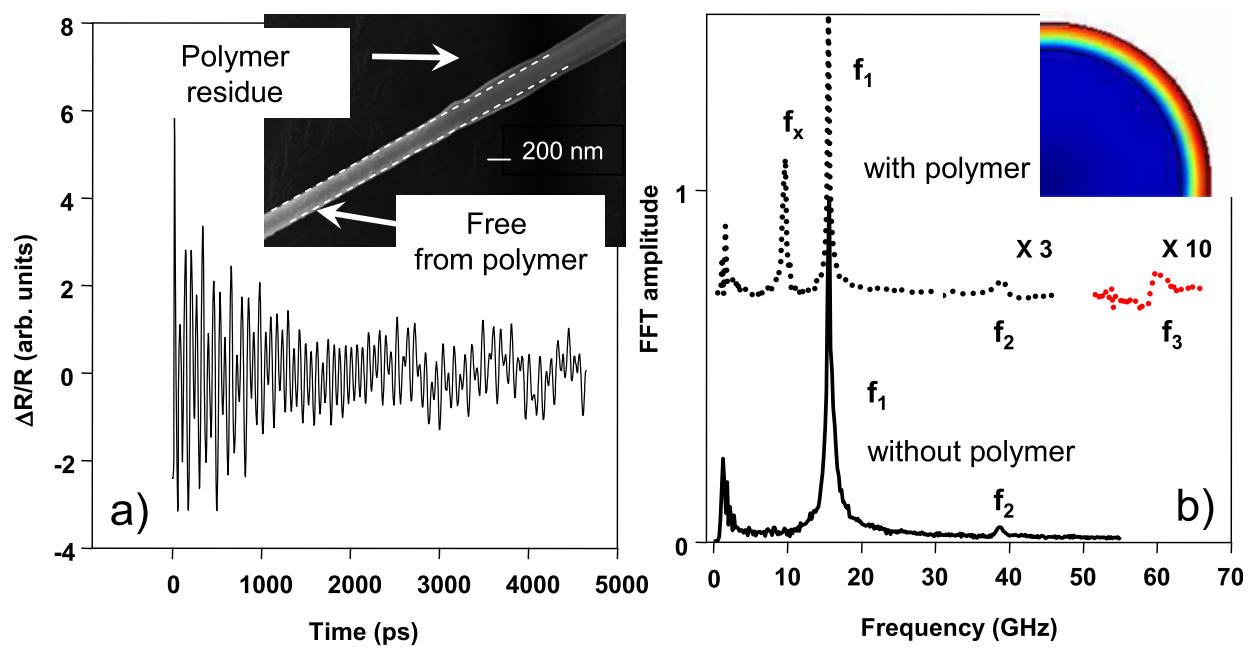

FIG. 5. (a) Transient sample reflectivity obtained on a single free standing nanowire with a diameter of $200 \mathrm{~nm}$. (b) Comparison of Fast Fourier transforms for a free standing wire embedded in polymer residue and for a wire free from any polymer cladding, previously located by electron microscopy. The weak and broad residual signature in the very low frequency domain (between 0 and $2 \mathrm{GHz}$ ) is not relevant with any vibrational signature but find its origin in the process to compute the FFT without any apodization function. This residual feature could be suppressed with generalized Hamming function. We can notice that signatures with frequency $\mathrm{f}_{\mathrm{x}}$ lower than $15.6 \mathrm{GHz}$, coming from vibrational modes into the cladding, are eliminated. Left inset: SEM picture presenting a wire with a clean surface and an area embedded in a polycarbonate shell. Right inset: Total displacement mapping of the first breathing mode ( 9 GHz) into the encapsulation shell for a thickness of $20 \mathrm{~nm}$ with $1.2 \mathrm{~g} / \mathrm{cm}^{3}, 2.1 \mathrm{GPa}$, and 0.38 for the density, the Young modulus, and the Poison ratio, respectively, of the MAKROFOL polymer. 
similar system in contact with a substrate, such high frequency vibrations are quenched. Nevertheless up to now, no clear breakdown has been experimentally revealed but further investigations on smaller wire diameters are in progress.

We studied a large number of free standing structures observing an extremely small fluctuation of the first breathing mode frequency value, namely less than $2 \%-3 \%$. In addition, for some structures we observed an additional feature placed usually at frequencies $f_{x}$ lower than the first mode (e.g., see $f_{x} \sim 9.2 \mathrm{GHz}$ in Fig. 5(b)). The position of this feature $\mathrm{f}_{\mathrm{x}}$ exhibited very large fluctuations, being located between $3 \mathrm{GHz}$ and $10 \mathrm{GHz}$. The origin of this signature cannot be explained by fluctuations of the wire diameter itself. Scanning electron microscopy (SEM) revealed in some cases a shell around the nanowire consisting of residual polycarbonate whose thickness varies significantly along the nanowire (Fig. 5(a), inset). 2D finite elements simulation predicts possible breathing modes into this polymer shell located between $\sim 3 \mathrm{GHz}$ and $\sim 10 \mathrm{GHz}$, for shell thicknesses between $50 \mathrm{~nm}$ and $15 \mathrm{~nm}$, respectively. These values are in good agreement with the polymer shell sizes revealed by SEM measurements. Time resolved experiments performed on selected wires, free from any polycarbonate residue, presented in Fig. 5(b) exhibit as expected only breathing modes signatures of the $\mathrm{Cu}$ nanowire.

Finally, we have investigated the dynamic response in the case of two free standing wires in close proximity as illustrated in the SEM image in Figure 6(a). Since the diameters of the two wires differ by less than $2 \%$, they resemble two almost identical oscillators, which when pumped and probed

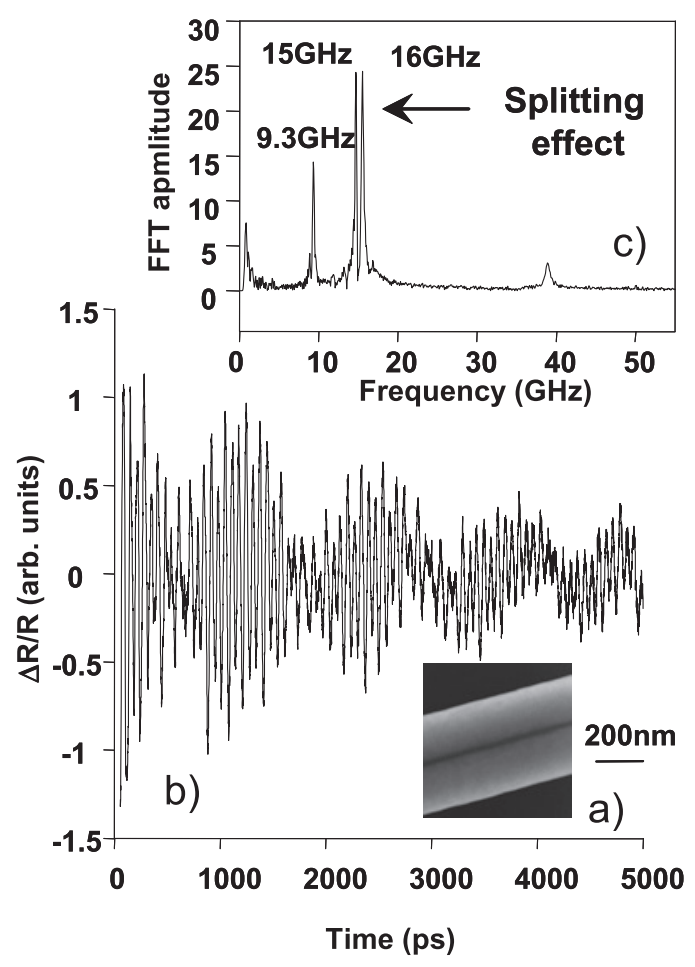

FIG. 6. Transient sample reflectivity obtained on two coupled copper nanowires. A clear beating phenomenon is observed, with two close frequency components in the Fast Fourier spectrum which is presented on the top. Inset: SEM picture presenting the two very close nanowires measured, exhibiting the same diameter. simultaneously should exhibit nearly the same breathing modes frequency. Figure 6(b) presents the dynamic response of these two wires exhibiting a beating structure which is revealed as well in the Fourier spectrum displayed in Fig. 6(c). Thus, the acoustic features corresponding to the first breathing mode are located at $15 \mathrm{GHz}$ and $16 \mathrm{GHz}$. Nevertheless the frequency splitting amplitude of the first breathing mode, obviously larger than spectral resolution, is around $1 \mathrm{GHz}$. In other words, the splitting effect is much larger than a simple discrepancy of the resonance pulsation of the free oscillators due to the small difference in size, estimated at $0.6 \mathrm{GHz}$. This phenomenon can be explained by a coupling between the two wires via polymer residues. The presence of coupling media between the wires is revealed as well by the lower acoustic feature around $9.3 \mathrm{GHz}$. In such case, a system of coupled equations for the two oscillators could be written as followed:

$$
\begin{aligned}
& \ddot{x}_{1}+\omega_{1}^{2} x_{1}=b_{1} x_{2} \\
& \ddot{x}_{2}+\omega_{2}^{2} x_{2}=b_{2} x_{1}
\end{aligned}
$$

with $\omega_{1}$ and $\omega_{2}$ being the resonance pulsation of the two oscillators without coupling, $b_{1}$ and $b_{2}$ being the coupling factors, and $\mathrm{x}_{1}$ and $\mathrm{x}_{2}$ being the motions of the two oscillators.

From this set of equations, the two eigenmode pulsations of the system formed by the two wires may be deduced,

$$
\begin{aligned}
& \omega_{f 1}=\sqrt{\frac{\omega_{1}^{2}+\omega_{2}^{2}-\sqrt{4 b_{1} b_{2}}}{2}} \text { and } \\
& \omega_{f 2}=\sqrt{\frac{\omega_{1}^{2}+\omega_{2}^{2}+\sqrt{4 b_{1} b_{2}}}{2}}
\end{aligned}
$$

Consequently, for a finite coupling efficiency, a global response composed by two frequency components slightly splitted around the initial frequency resonance of the single oscillators is predicted. For the present case, this frequency amounts to around $15.6 \mathrm{GHz}$ being in good agreement with the results reported in Figure 6(c). We estimated the spring constant which can describe the coupling across the polymer using the splitting amplitude, and compared it to ES/L where $\mathrm{E}$ is the Young modulus of the polymer, $\mathrm{S}$ is the surface of contact area, and $\mathrm{L}$ is the gap distance between the two nanowires. A value of $10 \mathrm{~nm}$ could be estimated from the SEM images for the gap nanowire distance. Taking into account an encapsulation shell thickness of $20 \mathrm{~nm}$ the contact surface is equal to $75^{*} 1 \mathrm{~nm}^{2}$ where 1 is an arbitrary length along the growth axis. In spite of such a simple model, the measured splitting effect can be reproduced assuming a Young modulus for the coupling media of $\sim 8 \mathrm{GPa}$ which is not so far from the value of the MAKROFOL polymer $(2.1 \mathrm{GPa})$ given by the manufacturer. Such fine effect, across the polycarbonate cladding, could not be revealed for a system which is in interaction with the substrate, because of a poor spectral resolution. For the same reason, it is also illusory to estimate the substrate influence on the frequency position of the breathing mode of vibration in such a local oscillator. 


\section{CONCLUSIONS}

In summary, we have shown that thanks to the use of free standing copper nanowires, we were able to observe different orders of the breathing mode up to $78 \mathrm{GHz}$. We obtained a very good agreement between experimental and simulated frequencies in an isotropic copper assumption. Taking advantage of the suppression of the mechanical coupling between the nanostructure and the substrate, we have obtained nanooscillators exhibiting high quality factors. Finally, the elastic confinement gives us the unique opportunity to observe the vibrational response of two coupled wires embedded in polymer shell. In the future, such fine spectral features could provide a new way to extract subtle fluctuation of crystallographic orientation along a single nanowire and could be directly correlated with X-Ray diffraction techniques.

${ }^{1}$ S. A. Maier, M. L. Brongersma, P. G. Kik, S. Meltzer, A. A. G. Requicha, and H. A. Atwater, Adv. Mater. 13, 1501-1505 (2001).

${ }^{2}$ E. Chang, A. S. Sorensen, E. A. Demler, and M. D. Lukin, Nature Phys. 3, 807-812 (2007).

${ }^{3}$ C. Novo, A. M. Funston, and P. Mulvaney, Nature Nanotechnol. 3, 598 (2008).

${ }^{4}$ L. R. Hirsch, R. J. Stafford, J. A. Bankson, S. R. Sershen, B. Rivera, R. E. Price, J. D. Hazle, and N. J. Halas, J. L. West. Proc. Natl. Acad. Sci. USA 100, 13549 (2003).

${ }^{5}$ X. H. Huang, I. H. El-Sayed, W. Qian, and M. A. El-Sayed, J. Am. Chem. Soc. 128, 2115 (2006).

${ }^{6}$ M. A. Van Dijk, M. Lippitz, and M. Orrit, Phys. Rev. Lett. 95, 267406 (2005).

${ }^{7}$ J. Burgin, P. Langot, N. Del Fatti, F. Vallée, W. Huang, and M. A. El-Sayed, J. Phys. Chem. C 112, 11231 (2008).

${ }^{8}$ H. Staleva and G. V. Hartland, Adv. Funct. Mater. 18, 3809 (2008).

${ }^{9}$ R. Marty, A. Arbouet, C. Girard, A. Mlayah, V. Paillard, V. K. Lin, S. L. Teo, and S. Tripathy, Nano Lett. 11, 3301 (2011).

${ }^{10}$ T. A. Kelf, Y. Tanaka, O. Matsuda, E. M. Larsson, D. S. Sutherland, and O. B. Wright, Nano Lett. 11, 3893-3898 (2011).
${ }^{11}$ H. Staleva and G. V. Hartland, J. Phys. Chem. C 112, 7535 (2008).

${ }^{12}$ P. Zijlstra, A. L. Tchebotareva, J. W. M. Chon, M. Gu, and M. Orrit, Nano Lett. 8, 3493 (2008)

${ }^{13}$ P. M. Jais, D. B. Murray, R. Merlin, and A. V. Bragas, Nano Lett. 11, 3685 (2011)

${ }^{14}$ V. Juvé, A. Crut, P. Maioli, M. Pellarin, M. Broyer, N. Del Fatti, and F. Vallée, Nano Lett. 10, 1853 (2010).

${ }^{15}$ C. Bainier, C. Vannier, D. Courjon, J.-C. Rivoal, S. Ducourtieux, Y. De Wilde, L. Aigouy, F. Formanek, L. Belliard, P. Siry, and B. Perrin, Appl. Opt. 42, 691 (2003).

${ }^{16}$ P. Siry, L. Belliard, and B. Perrin, Acta. Acust. Acust. 89, 925 (2003).

${ }^{17}$ A. Vertikov, M. Kuball, A. V. Nurmikko, and H. J. Maris, Appl. Phys. Lett. 69, 2465 (1996).

${ }^{18}$ T. Bienville, J. F. Robillard, L. Belliard, I. Roch-Jeune, A. Devos, and B. Perrin, Ultrasonics 44, e1289 (2006).

${ }^{19}$ Y. Guillet, B. Audoin, M. Ferrié, and S. Ravaine, Phys. Rev. B 86, 035456 (2012).

${ }^{20}$ Y. Guillet, C. Rossignol, B. Audoin, G. Calbris, and S. Ravaine, Appl. Phys. Lett. 95, 061909 (2009).

${ }^{21}$ M. Hu, X. Wang, G. V. Hartland, P. Mulvaney, J. P. Juste, and J. E. J. Sader, Am. Chem. Soc. 125, 14925-14933 (2003).

${ }^{22}$ A. Amziane, L. Belliard, F. Decremps, and B. Perrin, Phys. Rev. B 83, 014102 (2011).

${ }^{23}$ C. Voisin, D. Christofilos, N. Del Fatti, and F. Vallée, Phys. B. Condens. Matter 316-317, 89-94 (2002).

${ }^{24}$ H. Y. Liang, M. Upmanyu, and H. C. Huang, Phys. Rev. B 71, 241403 (2005).

${ }^{25}$ H. Petrova, J. Pérez-Juste, Z. Y. Zhang, J. Zhang, T. Kosel, and G. V. J. Hartland, Mater. Chem. 16, 3957-3963 (2006).

${ }^{26}$ M. E. Toimil Molares, V. Buschmann, D. Dobrev, R. Neumann, R. Cholz, I. U. Schuchert, and J. Vetter, Adv. Mater. 13, 62 (2001).

${ }^{27}$ J. L. Duan, T. W. Cornelius, J. Liu, S. Karim, H. J. Yao, O. Picht, M. Rauber, S. Muller, and R. J. Neumann, Phys. Chem. C 113, 13583 (2009).

${ }^{28}$ L. J. Pochhammer, Reine Angew. Math. 81, 33-61 (1876).

${ }^{29}$ C. Chree, Trans. Cambridge Philos. Soc. 14, 250-369 (1889).

${ }^{30}$ E. Anthony Armenàkas, C. Denos Gazis, and G. Herrmann, Free Vibrations of Circular Cylindrical Shells (Pergamon Press Ltd, 1969).

${ }^{31}$ E. Barthel, J. Phys. D: Appl. Phys. 41, 163001 (2008).

${ }^{32}$ T. A. Major, A. Crut, B. Gao, S. S. Lo, N. Del Fatti, F. Vallée, and G. Hartland, Phys. Chem. Chem. Phys. 15, 4169 (2013).

${ }^{33}$ N. Combe and L. Saviot, Phys. Rev. B 80, 035411 (2009). 